\title{
Does elevated urinary Dkkopf-3 level predict vulnerability to kidney injury during cardiac surgery?
}

\author{
Matthew B. Lanktree ${ }^{1}$, York Pei ${ }^{2}$ \\ ${ }^{1}$ Divsion of Nephrology, St. Joseph's Healthcare Hamilton, McMaster University, Hamilton, Ontario, Canada; ${ }^{2}$ Divsion of Nephrology, University \\ Health Network and University of Toronto, Toronto, Ontario, Canada \\ Correspondence to: York Pei, MD. 8N838, Toronto General Hospital, 585 University Ave., Toronto, ON M6G2N2, Canada. Email: York.pei@uhn.ca. \\ Provenance: This is an invited article commissioned by the Section Editor Dr. Linpei Jia (Department of Nephrology, Xuanwu Hospital of Capital \\ Medical University, Beijing, China). \\ Comment on: Schunk SJ, Zarbock A, Meersch M, et al. Association between urinary dickkopf-3, acute kidney injury, and subsequent loss of kidney \\ function in patients undergoing cardiac surgery: an observational cohort study. Lancet 2019;394:488-96.
}

Submitted Oct 24, 2019. Accepted for publication Nov 01, 2019.

doi: $10.21037 /$ atm.2019.11.12

View this article at: http://dx.doi.org/10.21037/atm.2019.11.12

Acute kidney injury (AKI) occurs in approximately one quarter of patients undergoing cardiac surgery and is associated with increased short-term and long-term mortality, as well as prolonged time for mechanical ventilation, intensive care, and hospitalization $(1,2)$. AKI is defined by a sudden decrease in glomerular filtration rate typically with reduced urine output over a time period of hours to days; many patients with AKI also require renal replacement therapy (RRT) including hemodialysis or continuous RRT. Recovery from AKI is variable, with kidney function returning to baseline in most patients, but more than $10 \%$ have persistent chronic kidney disease (CKD) and 2\% have persistent advanced CKD (2). Multiple mechanisms contribute to kidney injury during cardiac surgery include: renal hypoperfusion and ischemic injury due to hypotension; non-pulsatile perfusion on cardio-pulmonary bypass; bypass-induced hemolysis and hemodilution; tubular damage from free hemoglobin, nitric oxide depletion; rhabdomyolysis; free radical release; activation of inflammatory pathways; and other causes (i.e., nephrotoxic drugs and radiographic contrast) (3).

Creatinine is a normal breakdown product of muscle turnover cleared by the kidney at a constant rate under steady state; thus, it can be used to estimate kidney function. However, serum creatinine is a biomarker of kidney function but not in itself a pathogenic factor for AKI, and a detectable rise in serum creatinine often requires 2 to 3 days following AKI. Other preoperative clinical risk factors for AKI post-cardiac surgery include baseline glomerular filtration rate, proteinuria, congestive heart failure, angina, diabetes, anemia, type of surgery (coronary artery bypass graft plus valve surgery). However, none of these risk factors can predict AKI post-cardiac surgery with high accuracy. Thus, there is an urgent need to develop better predictors of AKI post-cardiac surgery (1).

Biomarkers such as serum cystatin C, kidney injury molecule-1 (KIM-1), neutrophil gelatinase-associated lipocalin (NGAL), Nephrocheck (combination of insulinlike growth-factor binding protein 7 and tissue inhibitor of metalloproteinases), liver-type fatty acid-binding protein, heart-type fatty acid-binding protein, and interleukin-18 have been tested for their utility in predicting AKI following cardiac surgery. For example, a recent study of 106 patients found that the combination of peak serum cystatin C and peak urine KIM1/creatinine ratio provided a high OR $(5.32$; $95 \%$ CI: $1.31-21.7)$ for predicting postoperative AKI (4). However, these findings were based on a relatively small sample size and had not been validated in another independent cohort of patients with similar clinical characteristics. Thus, the recent study by Schunk et al. provides a welcome addition of biomarker studies on AKI (5).

DKK3 (Dkkopf-3) is a secreted glycoprotein primarily expressed in vascular and neural tissue with roles in endothelial cell differentiation, angiogenesis, and atherosclerosis (6). Circulating DKK3 antagonizes 
intracellular $\mathrm{Wnt} / \beta$-catenin signalling via a surface receptor (6). DKK3 is expressed in renal tubular epithelial cells and urinary DKK3 concentration is correlated with the extent of interstitial fibrosis and tubular atrophy (7). Interestingly, DKK3 is over-expressed in cystic lining cells in patients with PKD1 mutations, and genetic variation in DKK3 is associated with chronic kidney dysfunction in autosomal dominant polycystic kidney disease (ADPKD) (8). Schunk et al. evaluated preoperative urinary DKK3 as a predictor of AKI post cardiac surgery (5). Patients were collected in an observational derivation cohort $(\mathrm{n}=744)$ with 820 days of median follow up, and as a validation cohort $(n=216)$ in the prospective multi-center remote ischemia preconditioning RenalRIP trial (9) with 90 days of median follow up. Preoperative urinary DKK3 to creatinine ratio $(>471 \mathrm{pg} / \mathrm{mg}$ ) was associated with subsequent AKI (OR 1.65, 95\% CI: 1.10-2.47, $\mathrm{P}=0.015$ ), even after adjustment for measured confounders including age, sex, body-mass index, hypertension, diabetes, smoking status, and baseline estimate glomerular filtration rate. Similar results were observed in the RenalRIP validation cohort (OR 1.94, 95\% CI: $1.08-3.47, \mathrm{P}=0.026)$. Higher preoperative urinary DKK3-to-creatinine ratio was also inversely associated with lower estimated glomerular filtration rate at hospital discharge and persistent CKD in both the derivation and validation cohorts.

Numerous randomized control trials and metaanalyses have generally concluded that remote ischemic preconditioning does not lead to a significant meaningful reduction in AKI incidence, but there has been unexplained heterogeneity in results (10). Details regarding the exact biological mechanism by which remote ischemic preconditioning would prevent AKI remains unclear. RenalRIP reported a $28 \%$ relative risk reduction of AKI with three cycles of a 4 min inflation of a blood pressure cuff to $200 \mathrm{mmHg}$ after induction of anesthesia (9). Consistent with observations from the derivation cohort, elevated urinary DKK3-to-creatinine ratio was associated with $\mathrm{AKI}$ and persistent $\mathrm{CKD}$ in the placebo subgroup, but not in the remote ischemic preconditioning subgroup. This observation raises the tantalizing hypothesis that remote ischemic preconditioning reduces the AKI risk predicted by elevated DKK3. However, caution is required as this was a post hoc subgroup analysis and the risk estimate confidence intervals overlap between the two groups. No post-surgical urinary DKK3 levels were measured and the effect of remote ischemic preconditioning on urinary DKK3 is unknown.

In contrast to an agnostic approach such as by using machine learning to predict AKI where the mechanisms underlying the risk are completely unknown (11), the current study employs a biological candidate approach in which a specific marker (DKK3) with defined biological properties was tested as a risk factor underlying AKI. Despite the promising results for urinary DKK3 in this study, it is unclear whether the association between high urinary DKK3 levels and increased risk of AKI is causal. Moreover, urinary DKK3 could also be a marker of vascular injury independent of the kidney. Future mechanistic studies using animal models may provide insights to address this question. Moreover, a causal role for urinary DKK3 in AKI and CKD could be evaluated with Mendelian randomization, whereby genetic predictors of urinary DKK3 concentration are tested for association with AKI and CKD (12). Ultimately, an intervention that alters kidney DKK3 expression would need to be developed and tested in a prospective randomized control trial.

As the timing of the nephrotoxic insults are generally known and prospectively documented, cardiac surgery is an ideal clinical setting to identify risk factors and to evaluate new therapies for AKI. This approach is exploited by the current study to provide a strong study design. Together with a large sample of patients for the discovery and validation cohorts, the findings of this study are promising and robust. However, it is unlikely that urinary DKK3 alone, with an increased OR of 1.65-1.94, is sufficiently to be used clinically to predict AKI with high accuracy. In future studies, it would be interesting to test whether postoperative urinary DKK3 levels would be a better predictor of AKI since the latter samples may incorporate additional prognostic information during the cardiac operation (i.e., ischemic insults). Additionally, testing urinary DKK3 levels in combination with other promising biomarkers may further improve its prognostic performance. Ultimately, improved risk stratification will facilitate the testing of novel therapeutic agents for AKI in clinical trials by enabling the selection of a more homogenous cohort of high-risk patients, thereby improving the power of the study while reducing the number of patients required.

\section{Acknowledgments}

None.

\section{Footnote}

Conflicts of Interest: The authors have no conflicts of interest 
to declare.

Ethical Statement: The authors are accountable for all aspects of the work in ensuring that questions related to the accuracy or integrity of any part of the work are appropriately investigated and resolved.

\section{References}

1. Pannu N, Graham M, Klarenbach S, et al. A new model to predict acute kidney injury requiring renal replacement therapy after cardiac surgery. CMAJ 2016;188:1076-83.

2. James MT, Pannu N, Hemmelgarn BR, et al. Derivation and External Validation of Prediction Models for Advanced Chronic Kidney Disease Following Acute Kidney Injury. JAMA 2017;318:1787-97.

3. Sriperumbuduri S, Clark E, Hiremath S. New Insights Into Mechanisms of Acute Kidney Injury in Heart Disease. Can J Cardiol 2019;35:1158-69.

4. Neyra JA, Hu MC, Minhajuddin A, et al. Kidney Tubular Damage and Functional Biomarkers in Acute Kidney Injury Following Cardiac Surgery. Kidney Int Rep 2019;4:1131-42.

5. Schunk SJ, Zarbock A, Meersch M, et al. Association between urinary dickkopf-3, acute kidney injury, and subsequent loss of kidney function in patients undergoing cardiac surgery: an observational cohort study. Lancet

Cite this article as: Lanktree MB, Pei Y. Does elevated urinary Dkkopf-3 level predict vulnerability to kidney injury during cardiac surgery? Ann Transl Med 2019;7(Suppl 8):S296. doi: 10.21037/atm.2019.11.12
2019;394:488-96.

6. Chen T, Karamariti E, Hong X, et al. DKK3 (Dikkopf-3) Transdifferentiates Fibroblasts Into Functional Endothelial Cells-Brief Report. Arterioscler Thromb Vasc Biol 2019;39:765-73.

7. Federico G, Meister M, Mathow D, et al. Tubular Dickkopf-3 promotes the development of renal atrophy and fibrosis. JCI Insight 2016;1:e84916.

8. Liu M, Shi S, Senthilnathan S, et al. Genetic variation of DKK3 may modify renal disease severity in ADPKD. J Am Soc Nephrol 2010;21:1510-20.

9. Zarbock A, Schmidt C, Van Aken H, et al. Effect of remote ischemic preconditioning on kidney injury among highrisk patients undergoing cardiac surgery: a randomized clinical trial. JAMA 2015;313:2133-41.

10. Deferrari G, Bonanni A, Bruschi M, et al. Remote ischaemic preconditioning for renal and cardiac protection in adult patients undergoing cardiac surgery with cardiopulmonary bypass: systematic review and metaanalysis of randomized controlled trials. Nephrol Dial Transplant 2018;33:813-24.

11. Tomašev N, Glorot X, Rae JW, et al. A clinically applicable approach to continuous prediction of future acute kidney injury. Nature 2019;572:116-9.

12. Sekula P, Del Greco MF, Pattaro C, et al. Mendelian Randomization as an Approach to Assess Causality Using Observational Data. J Am Soc Nephrol 2016;27:3253-65. 